\title{
Skin closure after arthroscopy utilizing a pull-out bow-tie subcuticular suture
}

\author{
Lori Ann Nacius \\ Cecilia Pascual-Garrido \\ Paul Sagiv \\ Omer Mei-Dan
}

Sports Medicine and Hip Preservation Service, Department of Orthopedics University of Colorado, USA

\section{Corresponding author: \\ Omer Mei-Dan}

Sports Medicine and Hip Preservation Service

Department of Orthopedics, University of Colorado

School of Medicine

12631 E 17 th Ave, Mailstop B202, Academic Office 1, Room 4602, Aurora, CO 80045, USA

E-mail: Omer.meidan@ucdenver.edu

\section{Summary}

Background: suturing techniques employed to close subcuticular surgical incisions are varied. Purpose: we present the "bow-tie" stitch, which is removed by pulling one side of the stitch with no need for sharp object stitch cutting. The stitch results in good approximation and scarring while enabling proper oozing. Methods: we have used this suture repeatedly for wound closure after hip and knee arthroscopy; its application to other superficial skin closures is easily appreciated. Results: this method of skin closure allows for ease of tying for the surgeon, aesthetically pleasing results for the patient, pain-free suture removal, no risk of suture knots becoming embedded in healing tissue, and decreased risk of infection and damage to skin, as instruments are not required for suture removal.

KEY WORDS: stich, arthroscopic, portals.

\section{Introduction}

Proper subcuticular suturing technique is a vital portion of any surgery where healing by primary intention is required. During arthroscopic surgery, 2-3 surgical puncture incisions are commonly made allowing the surgeon to access the patient's joint in order to investigative and treat intra-articular pathology. These skin portals are then sutured at the end of the procedure and the respective stitches are typically removed in clinic at a post op visit.

Goals of wound closure after hip arthroscopy include adequate cessation of soft tissue iatrogenic dead space and application of sufficient tensile support at the surgical incision to provide satisfactory strength to healing skin and subcuticular tissues. Minimizing the risks of bleeding and infection are also necessary, as the surgical incision can be a portal for bacteria if not satisfactorily approximated. Aesthetically pleasing outcomes as a result of precise approximation of wound edges during the suturing process include negligible scarring and minimal evidence of stitching, thus enhancing patient satisfaction ${ }^{1}$.

Suturing techniques employed to close subcuticular surgical incisions are varied. Closure of wounds that allow for secure apposition of the edges of the surgical puncture incision can be accomplished with a myriad of tried and true methods. These can include as little intervention as that provided by use of adhesive and steri-strips in cases of small incisions which are not expected to see any stress, to more extensive involvement whereby several sub-dermal to skin stitching layers are necessary.

Most arthroscopy incisions are closed with a regular nylon mattress stitch, which carries two disadvantages in this specific set up. The stitch needs to be removed using a blade, which can cause apprehension to the patient and potential irritation or injury to the skin while the sealing of the joint is at times too tight and thereby prevents the required oozing which drains the soft tissue from the arthroscopically introduced fluid ${ }^{2}$.

We present the "bow-tie" stitch, which is removed by pulling one side of the stitch with no need for sharp object stitch cutting. The stitch results in good approximation and scarring while enabling proper oozing. We have used this suture repeatedly for wound closure after hip and knee arthroscopy; its application to other superficial skin closures is easily appreciated. This technique meets the ethical standards of MTLJ ${ }^{3}$.

\section{Technique}

Materials needed for wound closure employing the pull-out bow-tie suture include a needle holder, thumb tissue forceps, and sterile 3 or 4-nylon non-absorbable suture with attached needle. The standard wound closure for arthroscopy involves suturing the subcuticular layer. Tissue forceps are utilized to gen- 
tly grasp the tissue and judge alignment and approximation of both skin edges. This allows proper placement of the first pass as the tissue is stabilized. The first pass is placed at the midpoint of the wound length and $4 \mathrm{~mm}$ perpendicular to the outside of the incision, in an outside-in fashion (Fig. 1A, B). If the incision is longer than $15 \mathrm{~mm}$ two such stitches can be placed at one-third and two-thirds distance. The needle tip penetrates the tissue and is pulled through the incision itself toward the surgeon; the next pass captures as much as possible of the opposing side's underlying sub dermal tissue and is sewn exactly opposite the first pass, (again at midpoint of the wound length) at the wound's skin edge, in an inside-out fashion (Fig. 1C, D). The needle is then turned 180degrees and continues in the opposite direction with the third pass penetrating the skin on the opposite (first) side from where the second pass was thrown (Fig. 1E). The third pass penetrates the skin in an inside-out fashion at midpoint between the wound edge and the initial pass (Fig. 1F, G). By pulling the suture gently but firmly, this pass precisely approximates the soft tissues so that the wound closes. The surgeon continues to gently pull the suture material leaving 5 $\mathrm{cm}$ length of the thread (Fig. $1 \mathrm{H}$ ).

In usual custom, the long end of the suture is then rotated around the needle holder's tip in a clockwise di- rection for two complete turns (Fig. 1I). The short end of the suture is grasped with the needle holder tip as close to the skin as possible, and a "byte" is pulled through, forming a loop, with care bow-tie taken to not pull the entirety of the short end through the pass but gently tightening the suture, parallel to the surgical incision (Fig. 1J). Next, the long end of the suture is rotated counter clockwise around the needle holder one time, and the distal aspect of the loop is grabbed by the needle holder once more, maintaining the loop as the suture material is tightened parallel to the incision with gentle yet sufficient force so as to not constrict the tissue but to allow adequate approximation of the wound edges (Fig. 1K).

The long (needle) side of the stitch is cut SHORT (5 $\mathrm{mm}$ from the bow-tie), while the other side (previously the short side or non-needle side) is cut LONG, leaving approximately $4 \mathrm{~cm}$ length of thread (Fig. 1L). This is a crucial step of the technique as the suture will be removed by pulling out the long side, making the length difference between the sides evident so there is no ambiguity as to which side should be pulled (Fig. 1M, P).

For removal of the suture, an alcohol swab or a Betadine swabstick and one's own hand are the only materials required. As with other skin stitches, the bowtie ideally will remain in place for no less than 12

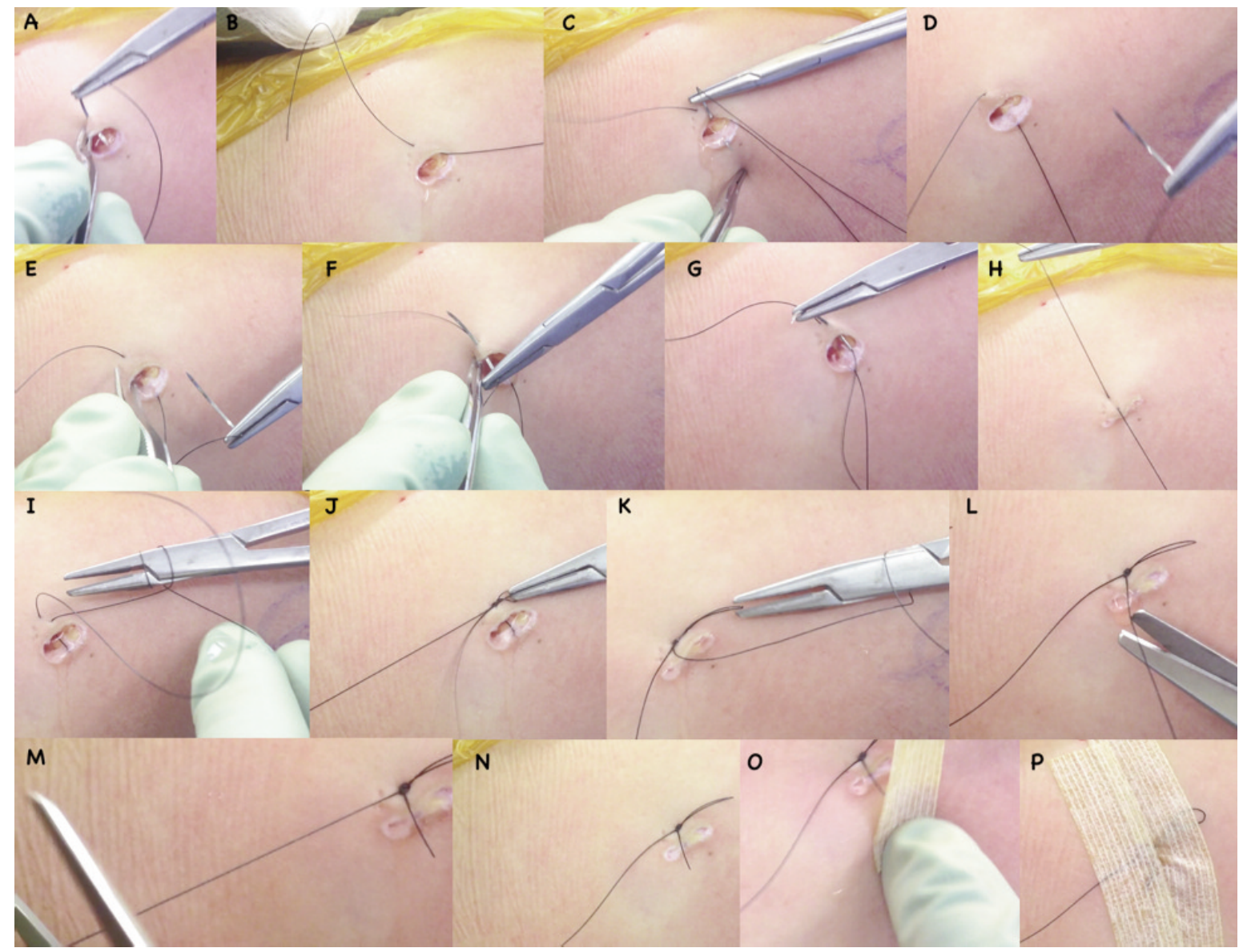

Figure 1. It shows step by step, how to perform the bow-tie knot. 

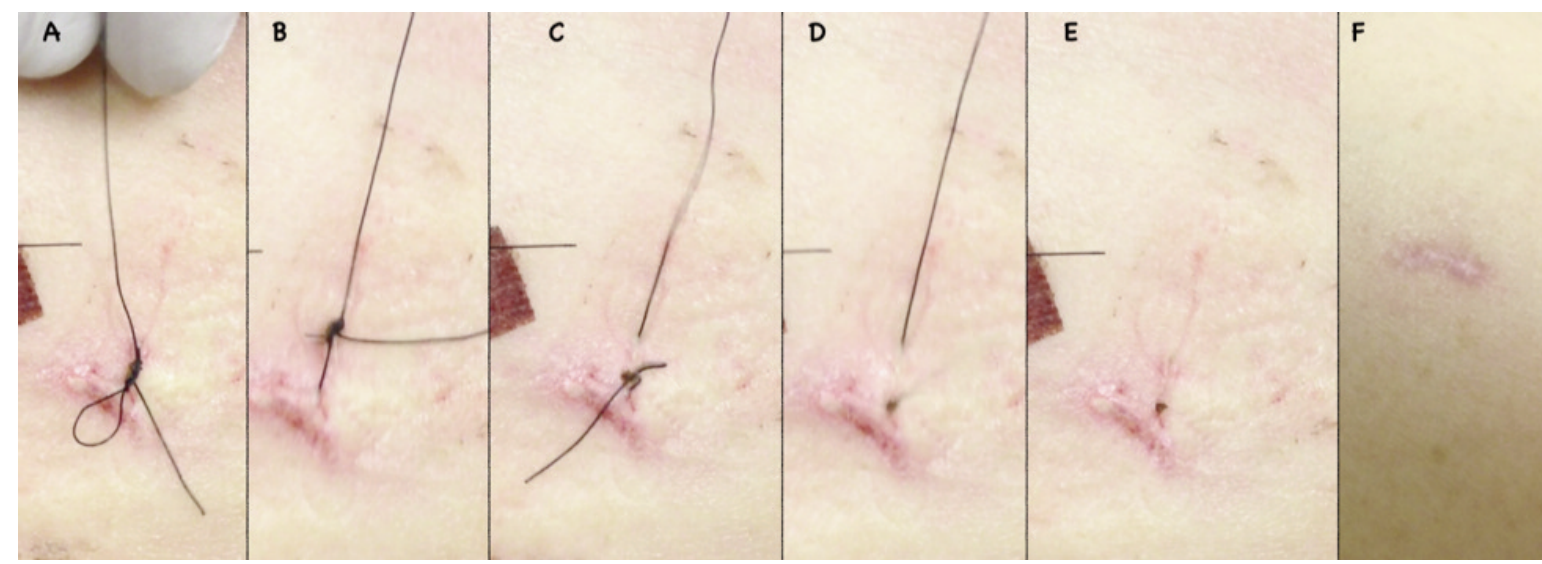

Figure 2. It shows step by step, how to remove the bow-tie knot.

days. Once skin healing is verified, the suture material and the healing wound are cleaned with an alcohol wipe or Betadine swabstick. The long end of the suture material is gently pulled and travels smoothly and easily through the skin; removal occurs without the need for scissors, cutting, or any manipulation of the suture material and can even be done by the patient himself in specific, necessary cases (Fig. 2 A-F). Steri-strips are then placed for a few more days; patients are instructed to allow these to fall off while in the pool or bath or to remove after 5 additional days.

\section{Discussion}

We have found the bow-tie suture to be superior to commonly used skin stitches due to the fact that it can be removed easily by staff who may not have experience with various suturing techniques. Additionally, the bow-tie results in good skin edges approximation and aesthetic scarring.

Many hip preservation and other high volume arthroscopic centers serve as referral centers and as so see many patients who travel from other states or countries, or drive many hours to be surgically treated. For these subjects, returning for a post op visit can be a real burden. The primary care physician or health care professional who will see these subjects in their first post op visit for wound check and stitch removal at times are not properly trained or familiar with skin suturing techniques and therefore, minimal interaction in this area is favorable. The bow-tie suture serves as an optimal tool in these cases.

Removal of the pull-out bow-tie suture is accomplished without the use of scissors or other surgical instruments and thereby prevents no risk of skin damage or nicking secondary to instrument slippage. On the surgeon's end, placement of the bow-tie suture is accomplished in a continuous, smooth fashion without the necessity for many repeated knots, which aim at preventing knot slippage. In addition, as opposed to most commonly used stitches, there is no risk of suture knots becoming embedded in healing skin.
Other commonly employed methods for skin closure include metallic staples and tissue adhesives; advantages and disadvantages exist for all choices. Staples offer a time-saving method for the surgeon in their application. However, staple removal has been shown to be more painful for the patient than removal of sutures while leaving significant skin scarring marks ${ }^{4}$. Tissue adhesives are another time-saving application, but can not sustain skin stress well while carrying added expensive compared to other methods of wound closure ${ }^{5}$. The proposed technique was written following the principles and recommendations for clinical research as proposed by Padulo et al.

\section{Conclusion}

Use of the pull-out bow-tie subcuticular suture is an effective way to close surgical puncture incisions after hip arthroscopy. This method of skin closure allows for ease of tying for the surgeon, aesthetically pleasing results for the patient, pain-free suture removal, no risk of suture knots becoming embedded in healing tissue, and decreased risk of infection and damage to skin, as instruments are not required for suture removal.

\section{References}

1. Smith TO, Sexton D, Mann C, Donell S. Sutures versus staples for skin closure in orthopaedic surgery: meta-analysis. BMJ. 2010; 340:1199-1203.

2. Edwards DJ, Elson RA. Skin closure using nylon and polydioxanone: a comparison of results. JR Coll Surg Edinb. 1995;40 (5):342-343.

3. Padulo J, Oliva F, Frizziero A, Maffulli N. Muscles, Ligaments and Tendons Journal. Basic principles and recommendations in clinical and field science research. MLTJ 2013;4:250-252.

4. Shantz JA, Vernon J, Leiter J, Morshed S, Stranges G. Sutures versus staples for wound closure in orthopaedic surgery: a randomized controlled trial. BMC Musculoskelet Disord. 2012:13:89-93.

5. Mashhadi SA, Loh CY. A knotless method of securing the subcuticular suture. Aesthet Surg J. 2011; (5):594-595. 